\title{
Current smoking improves ulcerative colitis patients' disease behaviour in the northwest of China
}

\author{
Huihong Zhai ${ }^{1}$, Wenyu Huang ${ }^{2}$, Aiqin Liü, Qianqian Li ${ }^{1}$, Qian Hao ${ }^{1}$, Ling Ma $^{1}$, Feng Yang ${ }^{1}$, Shutian Zhang \\ ${ }^{1}$ Department of Digestive Diseases, General Hospital of Ningxia Medical University, Yinchuan, China \\ 2Department of Digestive Diseases, Graduate School of Ningxia Medical University, Yinchuan, Ningxia, China \\ ${ }^{3}$ Department of Gastroenterology, Beijing Friendship Hospital Affiliated to Capital Medical University, Beijing, China \\ ${ }^{4}$ Beijing Key Laboratory for Precancerous Lesion of Digestive Diseases, Beijing, China \\ ${ }^{5}$ National Clinical Research Centre for Digestive Diseases, Beijing, China
}

Gastroenterology Rev 2017; 12 (4): 286-290

DOI: https://doi.org/10.5114/pg.2017.72104

Key words: current smoking, ulcerative colitis, Mayo score.

Address for correspondence: Shutian Zhang MD, PhD, Yongan St, Xicheng Area, 100050 Beijing, China, phone: +86 0106301441 ,

e-mail: zhangshutian@ccmu.edu.cn

\begin{abstract}
Introduction: The incidence of ulcerative colitis has increased tremendously over the past few decades, and caused much more attention in China. Several studies have revealed that both environmental and genetic factors play crucial roles in the formation and development of this disease. Among the environmental factors, the smoking condition is highly significant. Although current smoking has been associated with a protective effect on both the development and progression of ulcerative colitis (UC), the clinical characteristics of UC patients with current smoking in China is still unclear.

Aim: To clarify the relationship between current smoking and the UC in the northwest of China, patients diagnosed with ulcerative colitis in the General Hospital of Ningxia Medical University from 2003.03 to 2012.09 were assessed.

Material and methods: Data were collected about clinical features, cigarette smoking conditions, disease location, and therapeutic requirements. A total of 421 patients were included in this study, at diagnosis, 341 (81\%) patients were non-smokers, and the current smokers amounted to 80 (19\%).

Results: As for disease location, there were no differences between the current smokers and non-smokers regarding proctitis and extensive colitis, but the current smokers had less left-sided colitis than non-smokers $(p=0.049)$. Based on the Modified Mayo score, heavier smokers had lower scores than other smokers and non-smokers. For the therapeutic requirements, current smokers needed fewer corticosteroids than non-smokers ( $p=0.048)$.

Conclusions: We found that current smoking is closely associated with a protective factor for UC.
\end{abstract}

\section{Introduction}

Ulcerative colitis (UC) is a chronic idiopathic inflammatory bowel disease (IBD) and is now considered as a common cause of gastrointestinal disease in China [1]. Multiple studies have suggested that genetic susceptibility in combination with environmental factors compose a complex pathogenic system that causes a characteristic de-regulation of mucosal immunity $[2,3]$. Among those environment factors, the smoking condition is significantly meaningful [4]. Most retrospective studies from western countries have shown that current smokers with a diagnosis of UC are more likely to exhibit milder disease than ex-smokers and non-smokers, reduced rates of hospitalisation, colectomy, and need less fewer corticosteroids to clinically manage their disease [5]. However, due to the different geographical location, lifestyle, and genetic susceptibility, the manifestation of UC in the western and eastern countries, especially in the northwest of China, may be different. Bearing this in mind, we conducted this study. A total of 421 patients diagnosed with UC in the General Hospital of Ningxia Medical University, Yinchuan, China from 2003.03 to 2012.09 (10 years) were enrolled in this study.

\footnotetext{
Aim

We investigated whether there are some correlations between current smoking and UC in the northwest of China.
} 


\section{Material and methods Study population}

Patients diagnosed with UC $(N=421,234$ male, 187 female, median age at diagnosis: 42.7 years) from March 2003 to September 2012 were identified from the IBD databases of the General Hospital of Ningxia Medical University, Yinchuan, China. Confirmation of diagnosis of UC was based on a combination of clinical features, endoscopic and histological features, and the exclusion of an infectious aetiology [6]. We collected patients' information by using case-note reviews on the demographic, epidemiological, and clinical features. We made every effort to exclude the patients who should be diagnosed with Crohn's disease (CD), indeterminate colitis, irritable bowel syndrome, and dysentery. Patients' case information was obtained from the case record. Smoking definitions across these studies were similar [7]. For the purpose of this study, we defined non-smokers as those patients who had never smoked or smoked fewer than seven cigarettes/week, and former smokers as those who had given up smoking at least 12 months before diagnosis, in agreement with previous studies [8]. Those patients who smoked during the period of UC diagnosis or within the previous year but who had quitted smoking for more than 12 months during the follow-up period were considered as quitters. In all the other situations patients were considered to be current smokers. We took in the definition of pack-year to mean, for example, that a patient who has smoked one packet (20 pieces) per day for 10 years equates to a smoking history of 10 pack-years. If the patient had smoked half of one packet (10 pieces) for 10 years, this patient's smoking history would equate to 5 pack-years.

\section{Data collection}

In order to evaluate the impact of the smoking situation on disease outcome, we only collected some major disease-related events including disease anatomy location and treatment with steroids, as well as the Modified Mayo score during the first diagnosis. To define the disease locations, we adopted the Montreal classification criteria [9] (E1 means disease located in the proctitis; E2 left-sided colitis; E3 extensive colitis). Therapeutic requirements (Chinese herbs, amino salicylates, and steroids) were also collected. The disease treatment information was collected based upon the medicine taken within the first three months after the first time of UC diagnosis. If patients took more than one medicine within the first 3 months, we only recorded the medicine that the patients took firstly and primarily. In this study, we used the Modified Mayo Score
(MMS) [10] to evaluate the severity of the disease. The MMS score system has been acknowledged by The Chinese Medical Association and currently is considered as a guideline for clinical evaluation of severity of UC. Moreover, we only recorded the first hospitalisation data for repeatedly hospitalised patients. This study was approved by the Ethical Committee of Ningxia Medical University General Hospital.

\section{Statistical analysis}

All data were stored on a Microsoft Access database. Descriptive statistics for the qualitative data are expressed as a percentage and the mean. We used $\chi^{2}$ test and Mann-Whitney test to evaluate the differences in baseline characteristics, and a $p$-value equal to or less than 0.05 was considered as significantly different. Kaplan-Meier method was used to evaluate major disease-related events between the current smokers and non-smokers for the first time since the disease diagnosis. We used the log-rank test to assert the effect of smoking situation in each event. For the use of the Modified Mayo score, we adopted 95\% confidence intervals (Cls) to evaluate the difference between current smokers and non-smokers. All statistical tests used two-sided testing and were performed using the SPSS 17.0 package for Windows.

\section{Results}

\section{Smoking situation and disease presentation}

We collected 421 patients for this study. For smoking situation at first disease diagnosis, 80 patients (19\%) were current smokers (median of 15 cigarettes/ day) and 341 (81\%) patients were non-smokers. With the non-smokers, 3 patients $(<1 \%)$ were former smokers (median time since quitting smoking until UC diagnosis was 18 months), and 338 patients were never smoking, as shown in the flow chart (Figure 1). Basic characteristics of the groups (current smokers vs. non-smokers) are shown in Table I, which indicates that there is no difference in respect of age at diagnosis and the patient's place of residence $(p>0.05)$.

\section{Disease location and therapeutic requirement associated with smoking status}

The Montreal classification [9] was used to identify the disease location at the first time of disease diagnosis. As shown in Figure 2, the inpatient rate of active-smoking with the advanced disease (from E1 to E3) was $54 \%, 33 \%$, and $13 \%$, respectively. For non-smokers, the rates of disease location from E1 to E3 were $43 \%$, 


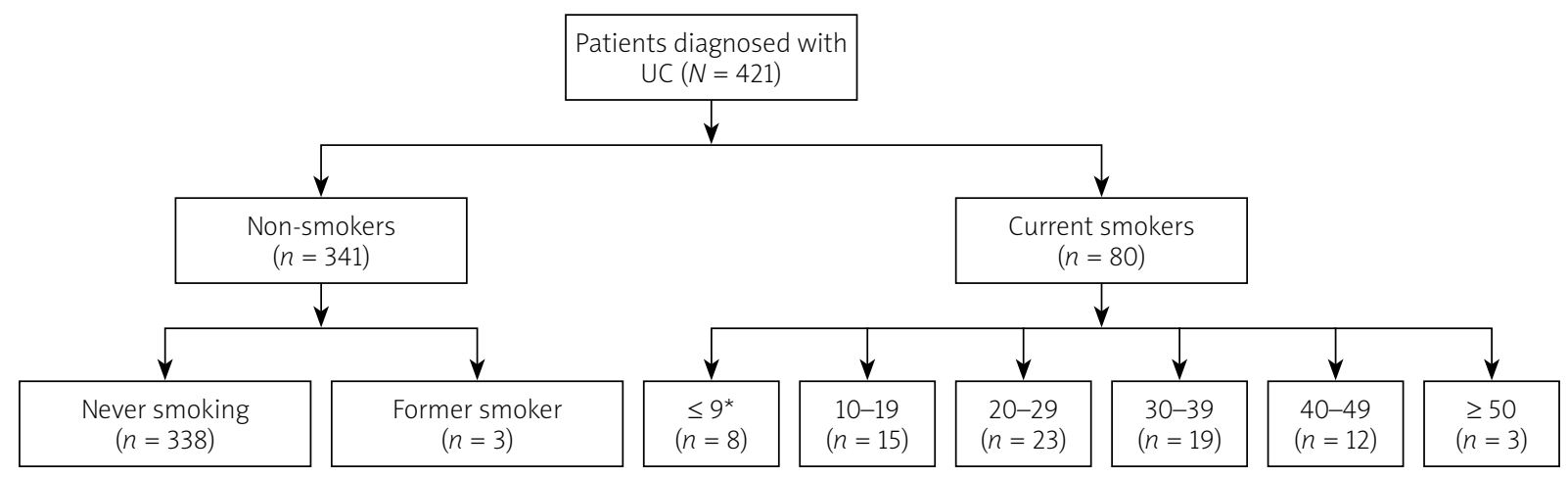

Figure 1. The flowchart of our research ( ${ }^{\star}$ means pack-years)

$45 \%$, and $12 \%$, respectively. In addition, for the disease location on the left-side (E2) their were fewer current smokers than non-smokers (33\% vs. 45\%, $p=0.049$ ). Therefore, we could draw the conclusion that current

Table I. Ulcerative colitis patients at diagnosis: demographic characteristics of patients according to the smoking status. Data presented in the brackets are percentages

\begin{tabular}{|c|c|c|c|}
\hline Parameter & $\begin{array}{c}\text { Non- } \\
\text { smokers } \\
n=341\end{array}$ & $\begin{array}{c}\text { Current } \\
\text { smokers } \\
n=80\end{array}$ & $P$-value* \\
\hline Age at diagnosis [years] & $39 \pm 12$ & $41 \pm 14$ & 0.352 \\
\hline Gender (male/female) & $171 / 170$ & $63 / 17$ & - \\
\hline Environment, $n$ (\%): & & & 0.603 \\
\hline Rural & $168(49)$ & $42(53)$ & - \\
\hline Urban & $173(51)$ & $38(47)$ & - \\
\hline $\begin{array}{l}\text { Disease extent } \\
\text { at diagnosis: }\end{array}$ & & & 0.120 \\
\hline Proctitis & $144(43)$ & $42(54)$ & 0.096 \\
\hline Left-sided & $152(45)$ & $26(33)$ & $0.049^{\star *}$ \\
\hline Extensive & $43(12)$ & $11(13)$ & 0.784 \\
\hline $\begin{array}{l}\text { UC therapy with the } \\
\text { first } 3 \text { month from } \\
\text { diagnosis, } n(\%) \text { : }\end{array}$ & & & 0.136 \\
\hline Chinese herb & $24(7)$ & $6(8)$ & 0.885 \\
\hline Amino salicylates & $165(49)$ & $48(60)$ & 0.062 \\
\hline Steroids & $152(44)$ & $26(32)$ & $0.048^{\star * *}$ \\
\hline Systemic application & $82(54)$ & $15(58)$ & - \\
\hline Local application & $70(46)$ & $11(42)$ & - \\
\hline
\end{tabular}

${ }^{*}$-value $\leq 0.05$ was considered significant different. ${ }^{* *}$ According to the Montreal's classification criteria, current smokers vs. non-smokers ( $33 \%$ vs. $45 \%, p=0.049)$. ${ }^{* * *}$ For the therapeutic requirements, current smokers vs. non-smokers ( $32 \%$ vs. $44 \%, p=0.048)$. smokers with the first-time diagnosis of UC are more likely to exhibit milder disease than non-smokers.

As shown in Figure 3, there were no significant differences between current smokers and non-smokers in the selection of medicine, including Chinese herbs and amino salicylates, but for the choice of steroids, among current smokers 26 (32\%) patients used steroids and among the non-smokers there were 152 (44\%) ( $p=0.048$, Figure 3$)$, which it means that current smokers need fewer corticosteroids and choose more mild medicine compared with other groups.

\section{Modified Mayo score associated with smoking status}

In this study, we adopted the Modified Mayo score, which was introduced in "The Consensus of Inflammatory Bowel Disease Diagnosis and Treatment (2012, Guangzhou, China)", and is widely used both in clinical scientific research and clinical activities. As shown in Figure 4, the scope of Modified Mayo score of patients who were non-smokers was 4-10, the mean value was approximately 8 , and the $95 \%$ confidence intervals (Cls) were 6-9, classified as moderate activity. Furthermore, for the current smoking patients, the scope of Modified Mayo score was 3-10, the mean value was approximately 7 , and the $95 \%$ confidence intervals (Cls) were $4.8-$ 8.3. These date show that there may be a trend in which more smoking will result in lower Modified Mayo score.

\section{Discussion}

We performed a retrospective analysis of data from The General Hospital of Ningxia Medical University, Yinchuan, China. Retrospective cohort studies of patients' smoking information were obtained prior to the diagnoses of UC. We found that current smoking is closely associated with a protective factor for UC. Current smokers with early diagnosis of UC are more likely to show milder disease and moderate need for the therapeutic requirements, need fewer corticosteroid, 
and choose milder medicine. Moreover, compared with non-smokers, current smoking patients with increasing pack-years showed lower Modified Mayo score.

Our findings regarding UC are consistent with observations reported by previous studies $[11,12]$. A research project conducted in the Ningxia province of China also found a similar result [13]. The risk of UC developing in current smokers compared with lifetime non-smokers is reported at an odds ratio (OR) of $0.41(95 \% \mathrm{Cl}$ : 0.34-0.48). Conversely, the risk of development of UC in lifetime non-smokers compared with current smokers has an OR of 2.9 (95\% Cl: 2.6-3.2) [14]. A Chinese research project conducted by the Chinese Inflammatory Bowel Disease Working Group in 2008 also found the same result, and they noticed that current smokers are less likely to develop UC than non-smokers (OR $=0.79$, 95\% Cl: 0.68-0.91, $p=0.001)$. From those research studies, we can learn that current smokers who have a diagnosis of UC are more likely to have milder disease than non-smokers with UC. Moreover, current smokers with UC have been found to have fewer relapses, fewer hospitalisations, and reduced need for corticosteroid and immunosuppressant therapy for their disease compared with non-smokers [15]. The biological mechanisms by which the smoking protects patients with UC are uncertain [16]. Based on the previous studies, some researchers believed that Heme oxygenase-1 (HO-1) and carbon monoxide (CO) in the smoking regulate intestinal homeostasis and mucosal immune responses to the enteric microbiota. $\mathrm{CO}$ and $\mathrm{HO}-1$ have been defined as a critically central homeostatic pathway in the intestine, which modulates the function of innate immunity, such as cytokine expression and macrophage bactericidal activity, and has been recently linked to the genetics and immunopathology of human $\operatorname{IBD}[17,18]$. There are also some reports showing that nicotine has some benefit for patients with active UC. Furthermore, studies have also demonstrated that cell apoptosis may be either decreased or increased in response to nicotine exposure $[19,20]$.

In contrast, some studies have demonstrated that there is no protective role of smoking for UC patients. For example, one study conducted in Asia-Pacific reported that in respect of the environmental risk factors for IBD [21] being breast fed for more than 12 months from birth, use of antibiotics, daily tea or coffee consumption, and the presence of a hot water tap and flushing toilet in childhood were protective for UC development, whereas there was no relationship between current smoking and the development of UC. Another research project conducted in China found almost the same results. They found that there was no negative association between the severity of

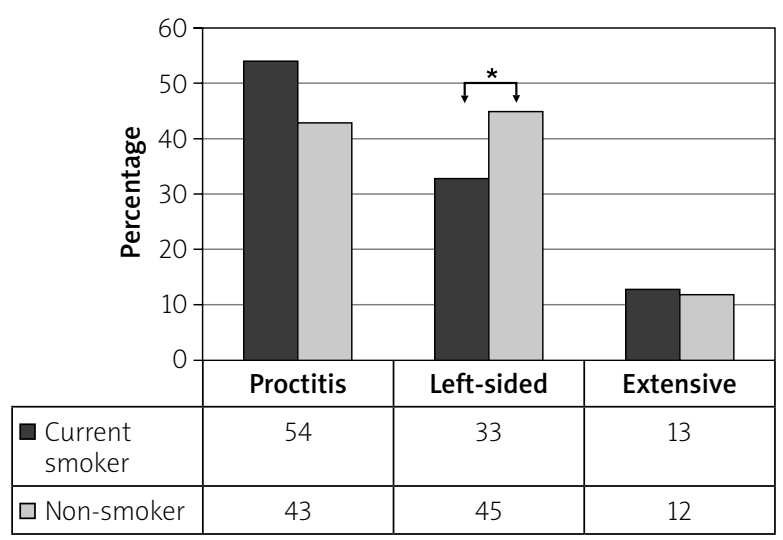

Figure 2. Disease location according to the smoking situations at the first diagnosis ( ${ }^{*}$ means $p$-value $\leq 0.05)$

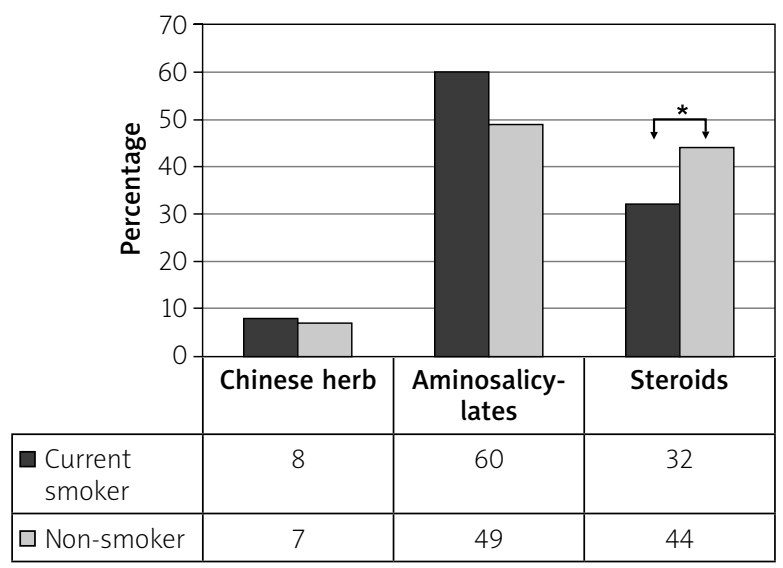

Figure 3. Ulcerative colitis therapy with the first 3 months of the first diagnosis ( ${ }^{*}$ means $p$-value $\leq 0.05)$

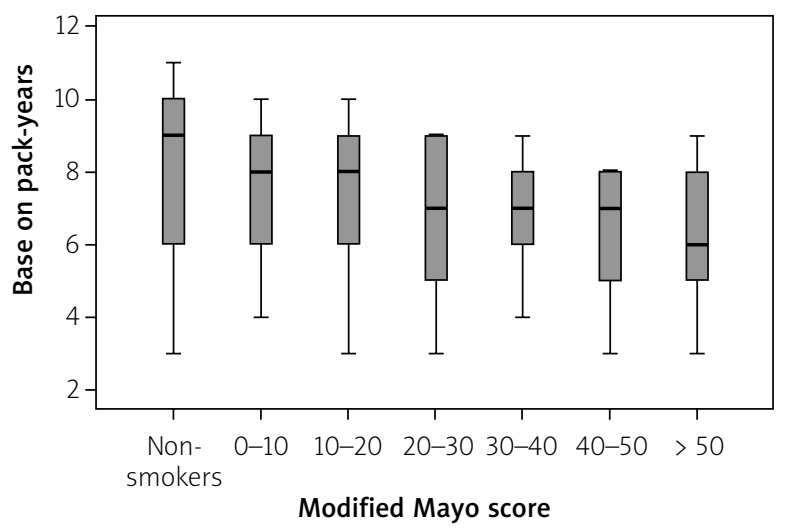

Figure 4. Modified Mayo Score according to the patients' smoking situation

UC and smoking $(p>0.05)$ [22]. The research conducted by Wang et al. investigating the risk factors of UC demonstrated that the protective factor for UC was 
tap water consumption, while the potential risk factors for UC were high sugar consumption, spicy food, heavy intake, and often feeling stress, and they did not find that current smoking had a significant correlation with the development of UC and the clinical features of the disease [20, 23].

Based on our current study, compared with other similar studies conducted in China, we recorded the UC patients' smoking conditions in a more detailed way, and retrospectively investigated the effect of smoking on disease behaviours, the need for therapy, and also the Modified Mayo score. However, because this is a single medical centre study, it is obvious that the result of our study cannot completely reflect the whole picture about the possible correlation of smoking and UC disease. Therefore, we will definitely work with other research centres to obtain a larger pool of patients, which hopefully will lead us to more meaningful data on the relationship between smoking and UC disease in the foreseeable future.

\section{Conclusions}

The incidence of UC has increased tremendously over the past few decades and has drawn a great deal of attention in China. In the northwest of China, current smokers with diagnosis of UC are more likely to have milder disease, and need fewer oral corticosteroids to manage their disease compared with non-smokers.

\section{Acknowledgments}

Huihong Zhai and Wenyu Huang contributed equally.

\section{Conflict of interest}

The authors declare no conflict of interest.

\section{References}

1. Ng SC. Epidemiology of inflammatory bowel disease: focus on Asia. Best Pract Res Clin Gastroenterol 2014; 28: 363-72.

2. Kaser A, Zeissing S, Blumberg RS. Genes and environment: how will our concepts on the pathophysiology of IBD develop in the future? Inflamm Bowel Dis 2010; 28: 573-621.

3. Baumgart DC, Carding SR. Inflammatory bowel disease: cause and immunobiology. Lancet 2007; 369: 1627-40.

4. Mahid SS, Minor KS, Soto RE, et al. Smoking and inflammatory bowel disease: a meta-analysis. Mayo Clin Proc 2006; 81: 1462-71.

5. Lunney PC, Leong RW. Review article: ulcerative colitis, smoking and nicotine therapy. Aliment Pharmacol Ther 2012; 36: 997-1008.

6. Ooi CJ, Fock KM, Makharia GK, et al. The Asia-Pacific consensus on ulcerative colitis. J Gastroenterol Hepatol 2010; 25: 453-68.

7. Zabana Y, Garcia-Planella E, Van Domselaar M, et al. Does active smoking really influence the course of Crohn's disease?
A retrospective observational study. J Crohn's Colitis 2013; 7: 280-5.

8. Chinese IBD Working Group. Consensus on the diagnosis and treatment of inflammatory bowel disease (Guang Zhou, 2012). Chin J Intern Med 2012; 17: 818-31.

9. Satsangi J, Silverberg MS, Vermeire S, et al. The Montreal classification of inflammatory bowel disease: controversies, consensus, and implications. Gut 2006; 55: 749-53.

10. D'Haens G, Sandborn WJ, Feagan BG, et al. A review of activity indices and efficacy end points for clinical trials of medical therapy in adults with ulcerative colitis. Gastroenterology 2007; 132: 763-86.

11. Ananthakrishnan AN. Epidemiology and risk factors for IBD. Gastroenterol Hepatol 2015; 12: 205-17.

12. Bastida G, Beltran B. Ulcerative colitis in smokers, non-smokers and ex-smokers. World J Gastroenterol 2011; 17: 2704-47.

13. Yanhong D, Jianfang L, Li Y, et al. Study on the ulcerative related risk factors in 239 cases. Ningxia Med J 2014; 36: 62-3.

14. Calkins BM. A meta-analysis of the role of smoking in inflammatory bowel disease. Dig Dis Sci 1989; 34: 1841-54.

15. Sun Y, Wang C. Smoking, nicotine and ulcerative colitis. Chin J Gastroenterol 2014; 19: 628-30.

16. Aldhous MC, Drummond HE, Anderson N, et al. Smoking habit and load influence age at diagnosis and disease extent in ulcerative colitis. Am J Gastroenterol 2007; 102: 589-97.

17. Altarescu G, Rachmilewitz D, Zevin S. Relationship between CYP2A6 genetic polymorphism, as a marker of nicotine metabolism, and ulcerative colitis. IMAJ 2011; 13: 87-90.

18. Onyiah JC, Sheikh SZ, Maharshak N, et al. Heme oxygenase-1 and carbon monoxide regulate intestinal homeostasis and mucosal immune responses to the enteric microbiota. Gut 2014; 5: 220-4.

19. Karban A, Elliakim R. Effect of smoking on inflammatory bowel disease: is it disease or organ specific? World I Gastroenterol 2007; 13: 2150-2.

20. Eliakim R, Karmeli F, Cohen P, et al. Dual effect of chronic nicotine administration: augmentation of jejunitis and aelioration of colitis induced by iodoacetamide in rates. Int I Colorectal Dis 2001; 16: 14-21.

21. Ng SC, Tang W, Leong RW, et al. Environmental risk factors in inflammatory bowel disease: a population-based case-control study in Asia-Pacific. Gut 2015; 64: 1063-71.

22. Wang YF, Ou-Yang Q, Xia B, et al. Multicenter case-control study of the risk factors for ulcerative colitis in China. World J Gastroenterol 2013; 19: 1827-33.

23. Jiang XL, Cui HF. An analysis of 10218 ulcerative colitis cases in China. World J Gastroenterol 2012; 8: 158-61.

Received: 21.05.2016

Accepted: 29.10 .2016 